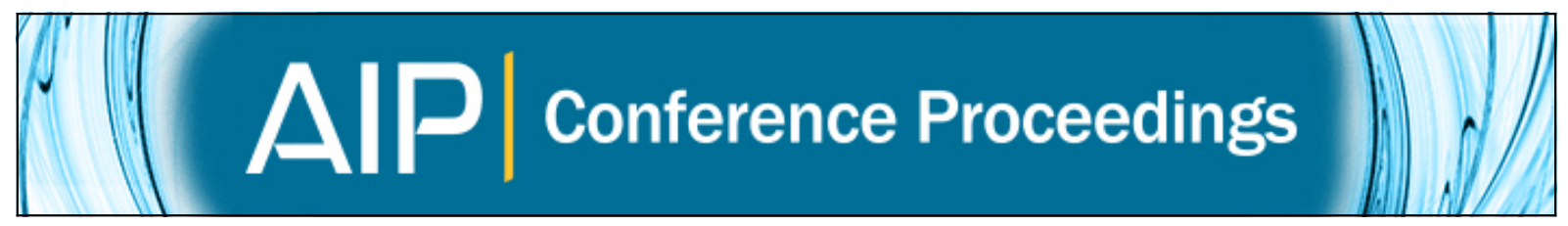

\title{
Solar energetic particle anisotropies and insights into particle transport
}

R. A. Leske, A. C. Cummings, C. M. S. Cohen, R. A. Mewaldt, A. W. Labrador, E. C. Stone, M. E. Wiedenbeck, E. R. Christian, and T. T. von Rosenvinge

Citation: AIP Conference Proceedings 1720, 070004 (2016); doi: 10.1063/1.4943841

View online: http://dx.doi.org/10.1063/1.4943841

View Table of Contents: http://scitation.aip.org/content/aip/proceeding/aipcp/1720?ver=pdfcov

Published by the AIP Publishing

\section{Articles you may be interested in}

Solar energetic particles: Shock acceleration and transport through self-amplified waves

AIP Conf. Proc. 1436, 212 (2012); 10.1063/1.4723610

Solar Energetic Particle Spectral Breaks

AIP Conf. Proc. 781, 227 (2005); 10.1063/1.2032701

Solar energetic 3 He mean free paths: Comparison between wave-particle and particle anisotropy results AIP Conf. Proc. 528, 165 (2000); 10.1063/1.1324304

Solar energetic particles: Acceleration and transport AIP Conf. Proc. 516, 103 (2000); 10.1063/1.1291471

Energetic particles in solar flares

AIP Conf. Proc. 56, 135 (1979); 10.1063/1.32074 


\title{
Solar Energetic Particle Anisotropies and Insights Into Particle Transport
}

\author{
R. A. Leske ${ }^{1, a)}$, A. C. Cummings ${ }^{1}$, C. M. S. Cohen ${ }^{1}$, R. A. Mewaldt ${ }^{1}$, A. W. \\ Labrador $^{1}$, E. C. Stone ${ }^{1}$, M. E. Wiedenbeck ${ }^{2}$, E. R. Christian ${ }^{3}$ and T. T. von \\ Rosenvinge $^{3}$
}

\author{
${ }^{1}$ California Institute of Technology, Pasadena, CA 91125 USA \\ ${ }^{2}$ Jet Propulsion Laboratory, California Institute of Technology, Pasadena, CA 91109 USA \\ ${ }^{3}$ NASA/Goddard Space Flight Center, Greenbelt, MD 20771 USA \\ a) ral@srl.caltech.edu
}

\begin{abstract}
As solar energetic particles (SEPs) travel through interplanetary space, their pitch-angle distributions are shaped by the competing effects of magnetic focusing and scattering. Measurements of SEP anisotropies can therefore reveal information about interplanetary conditions such as magnetic field strength, topology, and turbulence levels at remote locations from the observer. Onboard each of the two STEREO spacecraft, the Low Energy Telescope (LET) measures pitch-angle distributions for protons and heavier ions up to iron at energies of about 2-12 MeV/nucleon. Anisotropies observed using LET include bidirectional flows within interplanetary coronal mass ejections, sunward-flowing particles when STEREO was magnetically connected to the back side of a shock, and loss-cone distributions in which particles with large pitch angles underwent magnetic mirroring at an interplanetary field enhancement that was too weak to reflect particles with the smallest pitch angles. Unusual oscillations in the width of a beamed distribution at the onset of the 23 July 2012 SEP event were also observed and remain puzzling. We report LET anisotropy observations at both STEREO spacecraft and discuss their implications for SEP transport, focusing exclusively on the extreme event of 23 July 2012 in which a large variety of anisotropies were present at various times during the event.
\end{abstract}

\section{OBSERVATIONS}

The largest solar energetic particle (SEP) event detected so far in solar cycle 24 was observed at the Solar TErrestrial RElations Observatory (STEREO) [1] on 23 July 2012 [2, 3, 4] when the Ahead spacecraft was $121^{\circ}$ of heliolongitude westward from Earth. The active region source of this event was at $\sim \mathrm{W} 133^{\circ}$, over the limb from Earth but near central meridian from the viewpoint of Ahead, and the event was associated with an x-ray flare of estimated magnitude between M8.2 and X2.5 [5]. The shock or blast wave that hit STEREO-Ahead had a peak magnetic field strength of over $100 \mathrm{nT}$ and solar wind speed exceeding $2000 \mathrm{~km} / \mathrm{s}$ [2], and was followed by two interplanetary coronal mass ejections (ICMEs) [4]. Only a minor shock (with peak field strength of $\sim 10 \mathrm{nT}$ ) was detected at STEREO-Behind, $124^{\circ}$ of heliolongitude westward from Ahead; it is not clear if this was the flank of the tremendous shock seen at Ahead or associated instead with one of several other CMEs during this period [4]. Indeed, the ICME at Behind a day after the minor shock had the opposite chirality from those observed at Ahead, and therefore was unrelated [Y. Liu, private communication]. Although proton intensities at Ahead were comparable to the highest recorded near Earth since 1972 [3], intensities at Behind were almost 1000 times lower (Figure 1) as the source was beyond the east limb from this perspective. Interesting features were seen in the particle anisotropies at both STEREO spacecraft at various times during this event, as discussed below.

The Low Energy Telescope (LET) [6] onboard each STEREO spacecraft measures the composition, spectra, and time variations of SEPs from $\mathrm{H}$ through $\mathrm{Ni}$ at energies of about 2-50 MeV/nucleon. Sectored rates in 16 longitudinal viewing directions (averaging $\sim 12^{\circ}$ wide in longitude and covering $\pm 15-20^{\circ}$ of latitude about the ecliptic) are accumulated onboard for 10 different element and energy bands. Relative intensity spectrograms in Figure 2 illustrate the anisotropies observed by LET during the 2012 July event. Two broad bands represent particles arriving in the two LET 


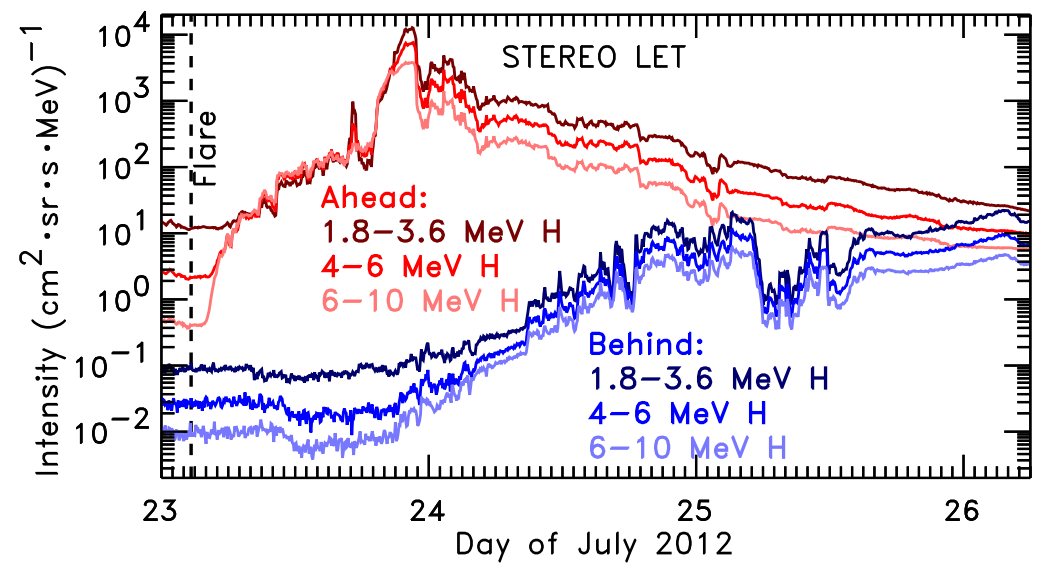

FIGURE 1. Time profiles at 5-minute resolution for omnidirectional $\mathrm{H}$ intensities in three energy bands from LET on STEREOAhead (red) and Behind (blue) during the July 2012 SEP event.

longitudinal viewing fans centered $45^{\circ}$ and $225^{\circ}$ from the Sun, with each fan comprising 8 sectors [6]; the white gap between the bands is due to coverage gaps in ecliptic longitude. When the count rate is very high, angular coverage for $\mathrm{H}$ and $\mathrm{He}$ in LET is deliberately restricted to reduce electronic deadtime [6], while coverage for heavy ions is unchanged, and this was the case at Ahead throughout much of this event. Therefore, although we use sectored rates of 4-6 MeV H for Behind in the bottom panel of Figure 2, we instead show 4-6 MeV/nucleon CNO sectored rates for Ahead in the top panel.

The most prominent feature in Figure 2 at Behind is seen during the first half of the indicated ICME. Since the peak intensity at this time appeared in the LET fan viewing away from the Sun, the energetic protons were flowing along the field line towards the Sun. In the opposite direction, particle intensities along the field were at a relative minimum, while in a narrow strip $180^{\circ}$ away from the edge of the incoming beam (at $\sim 20^{\circ}$ in Figure 2 ) intensities were elevated. Such a loss-cone distribution occurs when an incident particle distribution experiences partial mirroring at a magnetic field enhancement [7]. Particles at large pitch angles are reflected while those with smaller pitch angles pass through the constriction if the field is not strong enough to turn them around. This particular loss-cone distribution has been described in more detail in our earlier work $[8,9]$.

Throughout most of 24 July (after the shock passage at 20:55 on 23 July), the Ahead spacecraft was inside an ICME [4] and the particle flow was bidirectional (Figure 2) as is often observed in such structures [10], but intensities were higher in the direction facing away from the Sun, perhaps suggesting a shorter path to the particle source in this direction. Anisotropies subsided at both Ahead and Behind by late on 25 July (Figure 2) and proton intensities were nearly the same at both spacecraft (Figure 1), as the particles had isotropized throughout the quasi-trapped, expanding region behind the shock, as is often seen in large SEP events [11].

At the event onset at Ahead, while intensities allowed unrestricted angular coverage for $\mathrm{H}$ and $\mathrm{He}$, pitch-angle distributions were exponential and formed a field-aligned beam flowing outwards from the Sun. Curiously, although particle time intensity profiles were relatively smooth in the field aligned directions, at larger pitch angles the time profiles were not smooth and the intensities varied quasi-periodically, changing by factors of 5 or so on timescales of 10-15 minutes [12]. This rapid time variability of the pitch-angle distributions may be seen in the 1-minute samples shown in Figure 3, and appeared in all 3 sectored $\mathrm{H}$ rates (from 1.8-10 MeV) and both sectored He rates (from 4$12 \mathrm{MeV}$ /nucleon). Early in the event the beam width fluctuated repeatedly by $>25^{\circ}$ in a matter of minutes, while it was much steadier later in the event. Although the width was variable, the slope of the distribution seemed relatively constant, while the peak, field-aligned intensities seemed truncated with a restricted range of variability.

\section{DISCUSSION}

Within the 23 July event, as mentioned above, we have observed inward-flowing particles, a loss-cone distribution, bidirectional streaming, and a peculiar beam-width oscillation. Such observations can be used to help understand how particles become distributed throughout the inner heliosphere. 


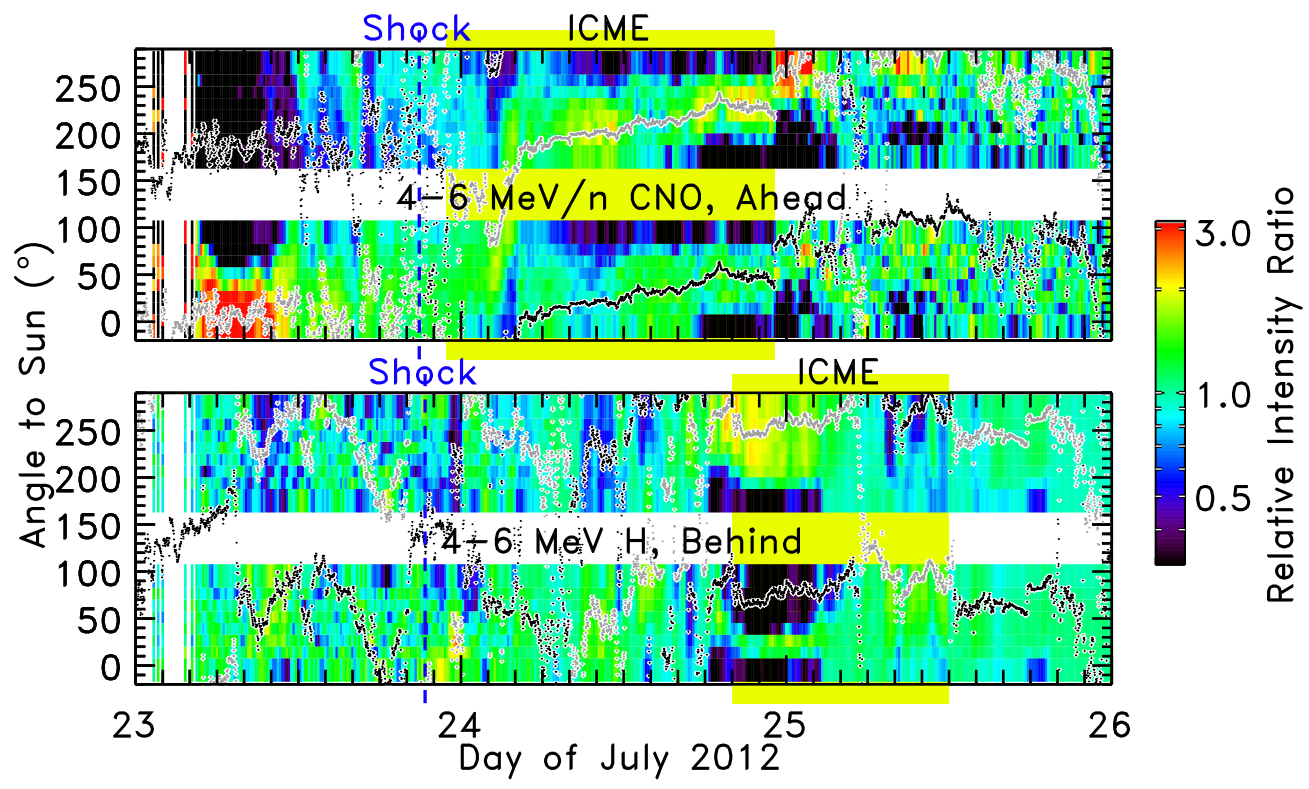

FIGURE 2. Relative sectored intensities in LET for 4-6 MeV/nucleon carbon through oxygen at STEREO-Ahead (top) and 4-6 MeV protons at Behind (bottom) during the July 2012 SEP event. At times the relative intensity ratios went significantly beyond the values of $\sim 0.3$ and 3 at which the color scale saturates (see Figure 3). Directions parallel (black) and antiparallel (gray) to the magnetic field longitudes measured by STEREO/MAG [13] are superposed. Vertical dashed lines and shaded regions indicate shocks and ICMEs, respectively, from http://www-ssc.igpp.ucla.edu/forms/stereo/stereo_level_3.html (however note that two distinct ICMEs were identified at Ahead in the shaded interval by [4]).

More details of the loss-cone distribution observed at Behind during this event were presented elsewhere [8, 9]. The loss-cone width depends on the ambient field strength and that at the mirror point [14, 15], which allows estimation of interplanetary conditions far from the observer. Although the spacecraft was inside an ICME at this time, the presence of the loss cone indicates that particles were injected into only one of the ICME legs, with partial mirroring occurring in the other. A loss cone would not have appeared sunward of the spacecraft in an undisturbed Parker spiral field, since eventually the field strength would have become large enough to mirror even the smallest pitch angles. Beyond the mirror point the field must have become weaker and allowed the smaller pitch-angle particles through, or particles must have been scattered onto other field lines. Perhaps interactions of the magnetic cloud with other CMEs or solar wind streams produced the necessary conditions, and comparison of the anisotropy observations with global heliospheric models may prove enlightening.

The oscillations in beam width at Ahead at the onset of the event are puzzling and remain to be explained. Interestingly, the magnetic field was nearly radial for more than a day, from midday on 22 July until the shock arrival late on 23 July, and radial fields facilitate the propagation of ion cyclotron waves due to reduced refraction and Landau damping in this geometry [16]. Lan Jian [private communication] reports intermittent ion cyclotron waves during the beam-width oscillation period, but they do not appear unusual. However, shortly before the arrival of the extreme shock (from 19:40-20:15), she finds several small flux ropes with some high frequency magnetic field fluctuations, which is rare. If these high frequency fluctuations had been present $\sim 13-16$ hours earlier when we observed the beam-width oscillations, and if they traveled at the ambient solar wind speed $(\sim 800 \mathrm{~km} / \mathrm{s}$ at 20:00 [2, 4]), they would have been located $\sim 0.27 \mathrm{AU}$ sunward of the spacecraft at 06:00, and $10 \mathrm{MeV}$ protons would have passed through them only $\sim 15$ minutes before we observed them. More work is needed to determine if the interaction of the particles with these field fluctuations might have produced the unusual anisotropy behavior. Meanwhile, it is also worth noting that by midday on 23 July particle intensities leveled off within a factor of $\sim 2$ of the streaming limit (Figure 1), which is caused by the scattering of particles by self-generated waves [17]. Although intensities were $\sim 10$ times lower during the oscillations, the restricted range of variability in the field-aligned intensities (Figure 3) suggests a possible relationship to the development of the streaming limit, or perhaps passage of the particles through self-generated waves upstream of the spacecraft. 


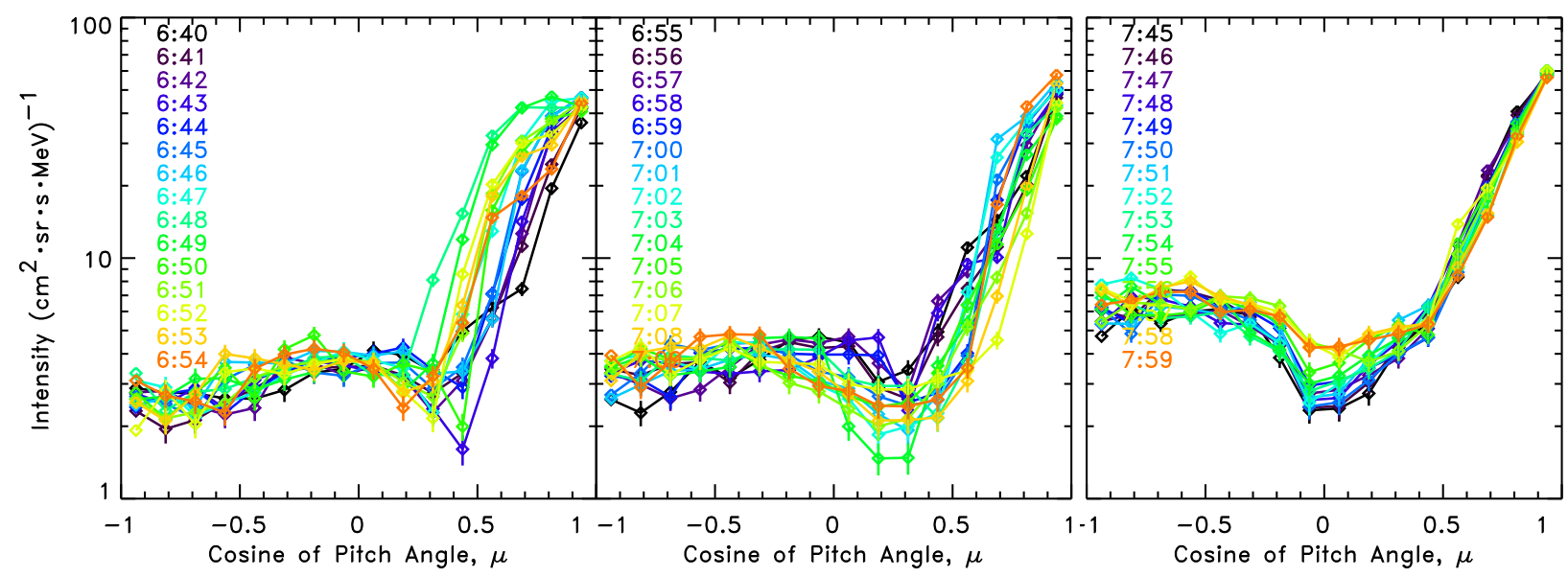

FIGURE 3. Sample one-minute averaged pitch-angle distributions for 6-10 MeV H in LET at STEREO-Ahead on 23 July 2012 at the times indicated.

\section{ACKNOWLEDGMENTS}

This work was supported by the National Aeronautics and Space Administration (NASA) at the California Institute of Technology and the Jet Propulstion Laboratory under sub-contract SA2715-26309 from the University of California at Berkeley under NASA contract NAS5-03131, and by NASA award NNX08AK87G. We thank the STEREO/MAG team for making their data publicly available, and Lan Jian and Ying Liu for helpful discussions.

\section{REFERENCES}

[1] M. L. Kaiser, T. A. Kucera, J. M. Davila, O. C. St. Cyr, M. Guhathakurta, and E. Christian, Space Sci. Rev. 136, 5-16 (2008).

[2] C. T. Russell, R. A. Mewaldt, J. G. Luhmann, et al., ApJ 770, p. 38 (2013).

[3] R. A. Mewaldt, C. T. Russell, C. M. S. Cohen, et al., Proc. 33rd Internat. Cosmic Ray Conf. (Rio de Janeiro), paper 1186 (2013).

[4] Y. D. Liu, J. G. Luhmann, P. Kajdič, et al., Nature Communications 5, p. 3481 (2014).

[5] N. V. Nitta, M. J. Aschwanden, P. F. Boerner, S. L. Freeland, J. R. Lemen, and J.-P. Wuelser, Solar Phys. 288, 241-254 (2013).

[6] R. A. Mewaldt, C. M. S. Cohen, W. R. Cook, et al., Space Sci. Rev. 136, 285-362 (2008).

[7] J. W. Bieber, W. Dröge, P. A. Evenson, et al., ApJ 567, 622-634 (2002).

[8] R. A. Leske, A. C. Cummings, C. M. S. Cohen, et al., "Observations of Loss-Cone Pitch Angle Distributions of Solar Energetic Particles," in Outstanding Problems in Heliophysics: From Coronal Heating to the Edge of the Heliosphere, Astronomical Society of the Pacific Conference Series, Vol. 484, edited by Q. Hu and G. P. Zank (2014), pp. 117-122.

[9] R. A. Leske, C. M. S. Cohen, R. A. Mewaldt, et al., Proc. 33rd Internat. Cosmic Ray Conf. (Rio de Janeiro), paper 0583 (2013).

[10] I. G. Richardson, V. M. Dvornikov, V. E. Sdobnov, and H. V. Cane, J. Geophys. Res. 105, 12579-12592 (2000).

[11] D. V. Reames, L. M. Barbier, and C. K. Ng, ApJ 466, 473-486 (1996).

[12] R. A. Leske, A. C. Cummings, C. M. S. Cohen, et al., Proc. 34th Internat. Cosmic Ray Conf. (The Hague), paper 072 (2015).

[13] M. H. Acuña, D. Curtis, J. L. Scheifele, C. T. Russell, P. Schroeder, A. Szabo, and J. G. Luhmann, Space Sci. Rev. 136, 203-226 (2008).

[14] K. A. Anderson, J. P. McFadden, and R. P. Lin, Geophys. Res. Letters 8, 831-834 (1981).

[15] L. C. Tan, D. V. Reames, C. K. Ng, O. Saloniemi, and L. Wang, ApJ 701, 1753-1764 (2009).

[16] L. K. Jian, C. T. Russell, J. G. Luhmann, R. J. Strangeway, J. S. Leisner, and A. B. Galvin, ApJL 701, L105-L109 (2009).

[17] D. V. Reames and C. K. Ng, ApJ 504, 1002-1005 (1998). 